\title{
Diagnosis and treatment of dementia: 6. Management of severe Alzheimer disease
}

\author{
Nathan Herrmann MD, Serge Gauthier MD
}

\section{ABSTRACT}

Background: The management of severe Alzheimer disease often presents difficult choices for clinicians and families. The disease is characterized by a need for full-time care and assistance with basic activities of daily living. We outline an evidence-based approach for these choices based on recommendations from the Third Canadian Consensus Conference on the Diagnosis and Treatment of Dementia.

Methods: We developed evidence-based guidelines using systematic literature searches, with specific criteria for the selection and quality assessment of articles, and a clear and transparent decision-making process. We selected articles published from January 1996 to December 2005 that dealt with the management of severe Alzheimer disease. Subsequent to the conference, we searched for additional articles published from January 2006 to March 2008 using the same search terms. We graded the strength of the evidence using the criteria of the Canadian Task Force on Preventive Health Care.

Results: We identified 940 articles, of which 838 were selected for further study. Thirty-four articles were judged to be of at least good or fair quality and were used to generate 17 recommendations. Assessment of severe Alzheimer disease should include the measurement of cognitive function and the assessment of behaviour, function, medical status, nutrition, safety and caregiver status. Management could include treatment with a cholinesterase inhibitor or memantine, or both. Treatment of neuropsychiatric symptoms begins with nonpharmacologic approaches to addressing behavioural problems. Severe agitation, aggression and psychosis, which are potentially dangerous to the patient, the caregiver and others in the environment, can be treated with atypical antipsychotics, with consideration of their increased risk of cerebrovascular events and death. All pharmacologic approaches require careful monitoring and periodic reassessment to determine whether continued treatment is necessary. Caregiver support and use of community resources are essential.

Interpretation: Severe Alzheimer disease requires frequent monitoring by health professionals. Simple nonpharmacologic approaches may address problems with mood and agitation. Antipsychotic drug therapy is occasionally necessary despite the inherent risks. Therapy with a cholinesterase inhibitor and memantine may be useful for selected patients.

Une version française de ce résumé est disponible à l'adresse www.cmaj.ca/cgi/content/full/179/12/1279/DC1

CMAJ 2008;179(12):1279-87

\section{The case}

You are caring for Mr. L, an 80-year-old man who had been given a diagnosis of Alzheimer disease 7 years ago. For the first 2 years, he was still able to drive, care for himself and live independently with his wife. His condition has deteriorated slowly over the past several years. At present, he is maintained at home with full-time help. He needs assistance to eat, dress and bathe.

Mrs. L, the primary caregiver, now finds him increasingly difficult to manage. She explains that her husband's periods of agitation, which were intermittent over the past 2 years, have recently been occurring several times a day, often triggered by viewing himself in the mirror. He has also started to talk to his reflection in the bathroom mirror. He occasionally has outbursts of aggressive behaviour. On one occasion he struck his wife after accusing her of having an affair with the person in the mirror. He is becoming resistant to being assisted with bathing and dressing.

Mr. L's score on the Mini-Mental State Examination is 9 out of 30. A therapeutic trial with a cholinesterase inhibitor was undertaken 2 years ago. The medication was stopped because cognitive decline continued rapidly, as indicated by caregivers' reports, scores on the Mini-Mental State Examination and consultation with a neurologist. Mr. L has a history of controlled hypertension. He is not taking other medications.

You ask how Mrs. L is coping with her husband's illness. She admits feeling very stressed. After additional questioning, she indicates that she has difficulty sleeping, constantly feels exhausted and has begun losing patience with her husband. How will you assist Mr. and Mrs. L?

$\mathrm{S}$ evere Alzheimer disease can be recognized when an individual needs full-time care and assistance with basic activities of daily living, such as bathing, dressing and toileting. Patients with severe Alzheimer disease will typically have a score of less than 10 on the Mini-Mental State Exami-

From the Department of Psychiatry (Herrmann), Sunnybrook Health Sciences Centre and University of Toronto, Toronto, Ont.; and the Alzheimer's Disease Research Unit, McGill Centre for Studies in Aging, and the Department of Neurology, McGill University (Gauthier), Montréal, Que.

This series is based on recommendations from the Third Canadian Consensus Conference on the Diagnosis and Treatment of Dementia.

Series editor: Howard Chertkow MD, Department of Neurology and Neurosurgery, McGill University, and the Bloomfield Centre for Research in Aging, Lady Davis Institute for Medical Research, Sir Mortimer B. Davis-Jewish General Hospital, Montréal, Que. 
Box 1: General approach to the management of patients with severe Alzheimer disease

- Patients should be monitored closely by health professionals at least every 4 months, or at least every 3 months if pharmacotherapy is used (grade C recommendation, level 3 evidence)

- Monitoring should involve assessment of the patients cognition, function, behaviour, and medical and nutritional status, as well as the caregiver's safety and health (grade B recommendation, level 3 evidence)

- The goals of management are to improve the quality of life for patients and caregivers, maintain optimal function and provide maximum comfort (grade B recommendation, level 3 evidence)

- Medical management includes treating intercurrent medical conditions (e.g., infections, parkinsonian symptoms, seizures, pressure ulcers), ameliorating pain, improving nutritional status and optimizing sensory function (grade B, level 3 evidence)

nation. The progression of the illness varies among individuals. Declines in cognitive scores do not always correlate with functional impairment. The median survival period from the diagnosis of Alzheimer disease is approximately 6 years (range 1-16 years), with about one-third of the time being spent in the severe stage. The Canadian Study of Health and Aging estimated that $50 \%$ of the people with Alzheimer disease in the study were in the moderate to severe stages of the illness at the time of diagnosis.

Because of their total dependence on others, patients with severe Alzheimer disease require much more caregiver time than those with milder dementia. The increased burden and stress on the caregiver often leads to the need for institutional care for the patient. In long-term care facilities, up to $90 \%$ of residents will have moderate to severe dementia.

Most practice guidelines for Alzheimer disease focus on the mild to moderate stages. Assessment tools and treatments that are applicable to earlier stages of the disease may not be as useful in the severe stage. Existing guidelines did not evaluate the studies of nonpharmacologic and pharmacologic therapies to improve cognitive function used specifically for this stage, which have only recently been published. Because of the prevalence of severe Alzheimer disease, the suffering of patients and their families, and the burden on society, the Third Canadian Consensus Conference on the Diagnosis and Treatment of Dementia has developed specific recommendations for the management of severe Alzheimer disease. ${ }^{2} \mathrm{We}$ present them in this article. A description of the process used to generate the recommendations is provided in the first article of the series ${ }^{3}$ and in Appendix 1 (available at www.cmaj.ca/cgi /content/full/179/12/1279/DC2).

\section{Assessment and general approach to management}

Patients with severe Alzheimer disease require close monitoring by health professionals. As the disease progresses to this stage, patients become more physically frail, their condition can change rapidly, and they experience more frequent and severe behavioural and psychological symptoms.

Family physicians should consider scheduling visits at least every 4 months, or at least every 3 months if pharmacotherapy is used. Monitoring should involve assessment of the patient's cognition, function, behaviour, and medical and nutritional status. Other important issues include assessments to determine the patient's risk of falls, other safety considerations, home modifications, community support services and eventually decisions concerning institutional care. During visits, health professional should also take the time to assess caregivers for physical and emotional distress (Box 1).

A frank and ongoing discussion with the caregiver regarding realistic goals of management at this stage of the illness should be undertaken. The main goals are to maximize the quality of life for the patient, maintain optimal function and provide maximum comfort. Having clearly stated advance directives and a plan for how to deal with emergencies is well advised. The plan should reflect the patient's wishes and expectations before the onset of severe disease.

\section{Patient assessment}

The Mini-Mental State Examination is recommended as a formal measure of cognitive function during routine visits. Although designed as a screening tool, the test should be used because of the familiarity of many physicians with it, its ease of administration, requirements by many provincial drug formularies to document its score as a criteria for reimbursement and the ability to compare this measure with outcome measures in trials of severe dementia. There are, however, concerns with the use of the Mini-Mental State Examination in assessing severe dementia. The scale suffers from "floor effects," becoming less sensitive to change as the disease progresses. More specifically, as the patient's condition continues to deteriorate, the test may not pick up clinically important changes.

A different scale, such as the Global Deterioration Scale (Box 2), ${ }^{4}$ may provide a better measure of overall severity. A score of 1 to 7 on the Global Deterioration Scale is assigned after a brief interview focused on the patient's needs for care and his or her function and behaviour. This step is followed by a brief examination of the patient's cognitive status. A score of 1 suggests no problems with activities of daily living, whereas a score of 7 indicates very severe cognitive decline, with the patient barely able to talk and unable to walk independently. Patients with severe Alzheimer disease have a score of 6 or 7 on the Global Deterioration scale. This scale is not widely used clinically and has not been fully validated as an outcome measure and monitoring tool for severe Alzheimer disease. As such, its limitations are not fully appreciated. ${ }^{5}$

Until further studies or other monitoring tools become widely available, we recommend that the Mini-Mental State Examination and the Global Deterioration scale be used in combination to monitor disease progression.

Besides performing a formal cognitive assessment of the 
patient, the physician should ask the caregiver about the patient's ability to communicate effectively, his or her ongoing recognition of family members, whether the patient gets lost within his or her home, and whether help with basic activities of daily living (e.g., dressing, bathing, feeding) are needed. Knowledge of a patient's bowel and bladder function is extremely important, because incontinence can be a crucial factor that leads caregivers to consider institutional care.

Behavioural and psychological symptoms of dementia become increasingly frequent and severe at this stage. Behavioural concerns include agitation, motor restlessness, aggression, apathy and psychosis (delusions and hallucinations). ${ }^{6}$ These symptoms are frequently associated with suffering for both the patient and the caregiver. They can also lead to concerns about patient and caregiver safety and can influence the need for early institutional care.

To monitor patients' medical status, health professionals should be vigilant for the detection of complications, including respiratory and urinary tract infections, seizures, pressure ulcers and parkinsonian symptoms. Increasingly, pain is recognized as an important concern and may be undertreated in patients with dementia. ${ }^{7}$ Ensuring the optimization of hearing

\section{Box 2: Global Deterioration Scale*}

Stage 1 No subjective memory deficit (no cognitive impairment); no problems with activities of daily living

Stage 2 Subjective memory complaints (subjective cognitive impairment): Complaints of being forgetful, such as complaints of trouble with recall of names; complaints of misplacing objects

Stage 3 Earliest clear deficits (mild cognitive impairment): Difficulties often noted at work; may have gotten lost; may have misplaced a valuable object

Stage 4 Clear deficits on clinical examination (moderate cognitive impairment): Decreased knowledge of personal and/or current events; often trouble with travel and finances

Stage 5 Can no longer survive independently in the community without some assistance (moderately severe cognitive impairment): Difficulty with recall of some important personal details (e.g., address, names of one or more important schools attended); may require cuing for activities of daily living

Stage 6 Largely unable to verbalize recent events in their life (severe cognitive impairment): May forget name of spouse; incontinence develops as this stage progresses; requires increasing assistance with activities of daily living; increased behavioural problems (e.g., agitation, delusions)

Stage 7 Few intelligible words or no verbal abilities (very severe cognitive impairment): Loses the ability to walk as this stage evolves

*Abridged version (c) Barry Reisberg MD, 2008. Modified from Reisberg $\mathrm{B}$, Ferris $\mathrm{SH}$, de Leon MJ, et al. The global deterioration scale for assessment of primary degenerative dementia. Am J Psychiatry 1982;139:1136-9. and vision may not only improve function but also have positive behavioural benefits. ${ }^{8}$ In addition to the medical status, health professionals should monitor patients' nutritional status. Treating malnutrition with nutritional supplements can be helpful, although many patients may simply require more help with feeding. ${ }^{9}$

Progressive gait instability, falls and parkinsonian symptoms are common features of severe dementia. Identification of impaired motor function and gait instability should lead to considerations for gait support such as canes and walkers.

A review of fall-related hazards at home should also be considered. Tools for the assessment of a patient's risk of falls have been developed for use in primary care. They include documenting the presence of previous falls, polypharmacy, history of stroke or parkinsonism, and reported balance problems as well as assessing the patient's difficulty getting out of a chair without the use of the arms (the "get up and go" test). ${ }^{10} \mathrm{~A}$ home assessment may result in the removal of small area rugs, the installation of railings and grab bars in washrooms, and the use of special locks and alarms to prevent wandering. Community-based occupational therapists can perform these in-home safety assessments.

Patients who are at risk of wandering should be registered with the Alzheimer Society of Canada's Safely Home Registry (www.safelyhome.ca/en/safelyhome/safelyhome.asp) and should wear a medical identification bracelet.

\section{Assessment of caregiver stress}

As part of regular monitoring, health professionals should assess the degree of stress experienced by caregivers. The heavy and continuous burden of care can have important psychological consequences, such as depression, feelings of hopelessness and anxiety; physical consequences, such as loss of sleep, appetite and energy, and pain; social isolation from an inability to spend time with friends or family or to engage in hobbies and other pleasurable pursuits; and financial strains because of direct costs of care as well as lost income. ${ }^{11,12}$

If any concerns arise during the evaluation, the caregiver should be directed to support services in the community, including those provided by the Alzheimer Society of Canada, daycare programs, respite care and home care. Education about the common features of severe dementia may provide some relief to friends and family. Caregivers may be guided to educational material from such agencies as the Alzheimer Society of Canada (www.alzheimer.ca/english/disease/progressionintro.htm). Should depressive symptoms be identified in caregivers, the physician should ask about the risk of suicide. If present, immediate action should be taken to ensure that the risks of self-harm are addressed by a trained health professional. If there is no risk of suicide, caregivers can be directed toward support programs for individual, family and group counselling, which have had positive, long-lasting benefits. ${ }^{13}$

\section{Consideration of institutional care}

Many patients with severe Alzheimer disease require fulltime institutional care. ${ }^{.16}$ Discussing the advantages and disadvantages of institutional care with caregivers is often challenging. Considering the patient's previously expressed 
wishes when competent is essential. However, caregivers often feel constrained by comments made years earlier and believe that the patient would not accept long-term care under any circumstances. It may be helpful to remind caregivers that earlier comments were made without a full appreciation of the current circumstances and that expectations almost always change with chronic illnesses. Circumstances that would not be tolerated when healthy are accepted once a person experiences the specific condition for an extended period. The patient's acceptance of the disabilities may be overshadowed by his or her feelings of dependence or feeling like a burden on others.

The advantages of long-term care should be emphasized. These include a structured environment and routine, the potential for increased stimulation, and the availability of nursing and medical supervision. Caregivers will often agonize over the decision and may be overcome by feelings of guilt. It can be helpful to involve other family members and other trusted health professionals, as well as to provide emotional support before, during and, most importantly, after the decision to move the patient to a long-term care facility.

Box 3: Management of behavioural and psychological symptoms associated with severe Alzheimer disease

- Nonpharmacologic interventions should be initiated first. Approaches that may be useful for severe Alzheimer disease include behavioural management for depression, and education programs for caregivers and staff to teach them how to recognize behavioural problems and to teach them behaviour-modification techniques. Music therapy and controlled multisensory stimulation (Snoezelen) are useful during treatment sessions, but longer-term benefits have not been demonstrated (grade B recommendation, level 1 evidence).

- Pharmacologic therapies should be initiated concurrently with nonpharmacologic interventions in the presence of severe depression, psychosis or aggression that puts the patient or others at risk of harm (grade B recommendation, level 3 evidence).

- Pharmacologic therapies for behavioural and psychological symptoms should be initiated at the lowest doses, titrated slowly and monitored for effectiveness and safety (grade B recommendation, level 3 evidence).

- Attempts to taper and withdraw medications for behavioural and psychological symptoms after a period of 3 months of behavioural stability should occur in a standardized fashion (grade A recommendation, level 1 evidence).

- Risperidone and olanzapine can be used for severe agitation, aggression and psychosis. The potential benefit of all antipsychotic agents must be weighed against the potential risks, such as cerebrovascular events and death (grade A recommendation, level 1 evidence).

- There is insufficient evidence to recommend for or against the use of trazodone in the management of agitated patients without psychosis (grade C recommendation, level 3 evidence).

- Benzodiazepines should be used only for short periods as needed (grade B recommendation, level 1 evidence).

\section{Management of behavioural and psychological symptoms}

When behaviours such as agitation, aggression and psychosis arise or worsen, it is important to search for the cause. They may be linked to worsening of the underlying neurodegenerative disorder. They may also be a due to delirium caused by an intercurrent medical condition (e.g., infection, vascular event or pain) or secondary to the effects of a new medication. ${ }^{17} \mathrm{~A}$ physical examination and review of medications should be performed. Laboratory investigations to rule out common causes of delirium include a complete blood count, measurement of serum electrolytes, calcium and fasting glucose, and urinalysis. The risk factors and precipitating factors for delirium have been reviewed in a previous article in this series. ${ }^{18}$

\section{Nonpharmacologic interventions}

Nonpharmacologic approaches should be considered before pharmacologic ones (Box 3). ${ }^{19}$ Although studies of nonpharmacologic interventions have rarely involved only people with severe Alzheimer disease, many treatments have been examined in Alzheimer disease in general. Systematic reviews ${ }^{19-23}$ have raised concerns about the methodologic rigour of many studies, but some therapies have been examined in randomized trials.

Behavioural treatment of depression is effective. ${ }^{24}$ Music therapy can alleviate agitation and apathy. ${ }^{25-27}$ Controlled multisensory stimulation, also known as Snoezelen, might help with apathy, and psychomotor therapy might alleviate agitation. ${ }^{21}$ Education and support programs for staff and caregivers are also effective. ${ }^{19}$ One benefit of support groups and counselling made available through the Alzheimer Society of Canada is that many creative strategies devised by individual caregivers can be shared with others.

Some nonpharmacologic interventions are widely available. However, others (e.g., Snoezelen) are available only in certain specialty care centres as part of daycare programs, or in long-term care facilities. Only some of the nonpharmacologic treatments have demonstrated lasting benefits (e.g., behavioural management and education programs for caregivers), whereas others (e.g., music therapy) appear to be effective only during treatment sessions. ${ }^{19}$

Despite the lack of strong, consistent evidence, the modest benefits, and questions about lasting benefit, nonpharmacologic interventions are recommended as first-line therapies, given the safety concerns related to pharmacologic therapies.

\section{Pharmacologic therapies}

Several randomized controlled trials have been published of atypical antipsychotic therapy for behavioural and psychological symptoms in patients with severe dementia. ${ }^{28}$ The studies suggest that risperidone at a dose of about $1 \mathrm{mg} / \mathrm{d}$ and olanzapine at a dose of 5-10 $\mathrm{mg} / \mathrm{d}$ are more effective than placebo. In the past few years, regulatory authorities, including Health Canada, have issued warnings that both of these drugs increase the risk of cerebrovascular events. ${ }^{29,30}$ They have also noted that all atypical antipsychotic agents increase the risk of death, according to findings from randomized controlled trials involving elderly patients with dementia. ${ }^{31}$ 
A meta-analysis of the randomized controlled trials on which the warnings were based suggested that the increased risk of cerebrovascular events was due primarily to nonspecific neurologic events rather than to completed strokes. ${ }^{32}$ Large observational studies have failed to confirm an increased risk of stroke among patients given atypical antipsychotics compared with patients given typical antipsychotics (e.g., haloperidol) or untreated patients. ${ }^{33,34}$

A meta-analysis of 15 randomized controlled trials evaluating dementia patients with behavioural and psychological symptoms concluded that there was an absolute risk difference of $1 \%(0.01,95 \%$ confidence interval [CI] 0.004-0.02; $p=0.01$ ) and an overall increase in the risk of death (odds ratio $1.54,95 \%$ CI $1.06-2.23 ; p=0.02$ ) with atypical antipsychotics compared with placebo. ${ }^{35}$ These findings are potentially worrisome. Alternatives such as haloperidol may have a risk of death that is at least comparable to, if not higher than, the risk associated with atypical antipsychotics. ${ }^{36-38}$

Since the Third Canadian Consensus Conference on the Diagnosis and Treatment of Dementia was held, several relevant studies of antipsychotic use have been published. In the Clinical Antipsychotics Trials of Intervention Effectiveness in Alzheimer Disease (CATIE-AD) — an important study of the pharmacologic treatment of psychosis, aggression and agitation in Alzheimer disease — 421 outpatients were randomly assigned to treatment with olanzapine, risperidone, quetiapine or placebo. ${ }^{39}$ The primary outcome measure was time to discontinuation of treatment for any reason. The results suggested no significant difference between any of the treatments compared with placebo.

Although these results are sobering, it is unclear whether they would have changed the recommendations of the consensus conference. Patients in the study had Mini-Mental State Examination scores of 5-26 (about 15 on average), which meant that they had moderate to severe Alzheimer disease, rather than only severe disease. These patients appeared to have less cognitive impairment and fewer behavioural problems than patients with severe Alzheimer disease in some of the randomized controlled trials of antipsychotic drugs.

Finally, the primary outcome measure - time to discontinuation of treatment for any reason - is not compatible with the recommendation that treatment be time-limited and that medication be withdrawn after a period of behavioural stability. In fact, results of the CATIE-AD study suggest that time to discontinuation of treatment because of lack of efficacy significantly favoured olanzapine and risperidone over placebo. The suggestion that atypical antipsychotics are more effective for patients with more severe behavioural disturbances is supported by findings from a recent meta-analysis of 4 randomized controlled trials of risperidone involving patients with Alzheimer disease. ${ }^{40}$

In an observational database study in the province of Ontario, Gill and colleagues ${ }^{38}$ examined the relation between antipsychotic use and mortality among patients with dementia. The results appeared to confirm the increased risk of death among patients given atypical antipsychotics compared with untreated patients. However, the risk of death with typical antipsychotics was even higher. The authors warned that their results may be diminished or eliminated by unmeasured confounding factors. One possible confounding factor was that the behaviours for which these drugs were prescribed may increase the risk for death, a relation that could not be properly assessed in the study.

The results of the study by Gill and colleagues ${ }^{38}$ strengthen the recommendations from the consensus conference about the need for careful consideration of the risks and benefits of atypical antipsychotic drugs. They also support the recommendation for the use of atypical antipsychotics only in the presence of severe agitation, aggression or psychosis that places the patient or those in their environment at risk.

Several randomized placebo-controlled studies, including those by Van Reekum and colleagues ${ }^{41}$ and Ballard and colleagues, ${ }^{42}$ have suggested that antipsychotics can be withdrawn in most patients without exacerbating behaviour. The consensus conference, therefore, recommends that clinicians consider tapering and withdrawing antipsychotics and all other medications used to treat behavioural and psychological symptoms after 3 months of behavioural stability.

Other psychotropic drugs might be useful for alleviating agitation and aggression. These include the antidepressants trazodone and citalopram as well as the anticonvulsant carbamazepine. ${ }^{43}$ On balance, the efficacy of the atypical antipsychotics appears to be superior to that of other classes of drugs despite the increased risks. ${ }^{28}$ However, there are few, if any, head-to-head comparisons to truly characterize all risks and benefits. Recently the American Psychiatric Association Practice Guidelines for the Treatment of Patients with Alzheimer's Disease and Other Dementias ${ }^{44}$ suggested that, on the basis of good evidence, antipsychotics should be recommended for the treatment of psychosis and agitation despite potentially serious adverse effects, with similar provisos about informed caregiver consent. Benzodiazepines have been studied in several trials. The best evidence for efficacy and safety was the short-term use of lorazepam for acute agitation. ${ }^{43}$ However, because of potential adverse events, including falls, excessive sedation, development of tolerance and worsening cognition, these agents should be used only for behavioural emergencies and as sedatives for procedures.

\section{Management of depression}

The assessment and diagnosis of depression in patients with a limited ability to communicate verbally can be challenging. The National Institute of Mental Health has devised provisional criteria for the diagnosis of depression in patients with Alzheimer disease. ${ }^{45}$ These criteria essentially modify those of the Diagnostic and Statistical Manual of Mental Disorders, Text Revision (DSM-IV-TR) for the diagnosis of major depressive episode. ${ }^{46}$ The significant differences include the requirement of fewer symptoms for the diagnosis (3 or more $\mathrm{v}$. 5 or more), less pervasiveness of symptoms, and the presence of irritability and social withdrawal or isolation.

The validity and reliability of the National Institute of Mental Health criteria, particularly in diagnosing depression in patients with severe Alzheimer disease, have yet to be established. If a patient is deemed to be severely depressed, 
Box 4: Management of depression in patients with severe Alzheimer disease

- Selective serotonin reuptake inhibitors can be used to treat severe depression (grade B recommendation, level 3 evidence)

- If behavioural disturbances fail to improve after appropriate nonpharmacologic and pharmacologic interventions, the patient should be referred to a specialty service (grade $B$ recommendation, level 3 evidence)

however, a physician should consider the option of treating the depression, even in the setting of severe Alzheimer disease (Box 4). ${ }^{43,47}$ Although most of the studies of the pharmacologic treatment of depression excluded patients with severe Alzheimer disease, the use of selective serotonin reuptake inhibitors is recommended, given their demonstrated efficacy and safety in patients with mild to moderate Alzheimer disease. . $3,47^{-}$

There are few data on the use of nonpharmacologic interventions for depression in patients with severe Alzheimer disease. ${ }^{19}$ One highly rated randomized trial concluded that the combination of exercise for patients and education of caregivers about behaviour-modifying techniques improved depression; however, it included patients with moderate to severe disease. ${ }^{48}$ The effects were modest, but benefits were noted in ratings of disability, depression, days of restricted activity and physical function. These benefits were evident throughout a 24-month follow-up period.

\section{Management of cognitive decline}

Pharmacologic therapies for the improvement of cognition include cholinesterase inhibitors and memantine (Box 5). Three randomized controlled trials of cholinesterase inhibitors involving patients with moderate to severe Alzheimer disease $^{49-51}$ and 2 randomized controlled trials involving only patients with severe Alzheimer disease ${ }^{52,53}$ suggested that this class of medication improves cognition, function, behaviour and global measures. The consensus conference recommends their use in patients with severe Alzheimer disease.

The most recent Cochrane review of cholinesterase inhibitors for Alzheimer disease ${ }^{54}$ was published before the 2 positive randomized controlled trials involving only patients with severe Alzheimer disease noted above. ${ }^{52,53}$ The review suggested that effects noted in patients with severe Alzheimer disease were similar to those in patients with mild to moderate dementia, although this statement was based on only 2 trials. ${ }^{49,50}$

Despite modest improvements in cognition and function, there is no evidence that cholinesterase inhibitors delay placement of patients in long-term care facilities.

Cardiac conduction defects, other than right bundle branch block, would be relative contraindications to the use of cholinesterase inhibitors. These drugs should be used with caution in patients who have severe chronic obstructive pul- monary disease and those with a history of peptic ulcer disease who are not taking cytoprotective agents. The most common side effects are gastrointestinal and include anorexia, nausea, vomiting and diarrhea.

Memantine can also be considered for the improvement of cognition. Five randomized controlled trials of memantine involved patients with severe Alzheimer disease or those with moderate to severe disease. ${ }^{55-58}$ One of the trials compared memantine and placebo in patients with severe disease who were taking donepezil. ${ }^{57}$ Several meta-analyses of these trials have suggested beneficial effects of memantine on cognitive, global and functional measures. ${ }^{59-61}$ Another meta-analysis of these trials suggested that the number needed to treat was 6 for the improvement or stabilization of global measures. ${ }^{62} \mathrm{~A}$ post-hoc analysis of 2 of the memantine studies suggested significant positive effects on agitation and aggression. ${ }^{63}$ However, there have been some anecdotal reports of worsening of behavioural symptoms with the use of memantine. ${ }^{64,65}$ Finally, in a Canadian pharmacoeconomic study, monotherapy with memantine was found to provide health benefits compared with standard care, without increasing costs. ${ }^{66}$ When deciding on therapies, physicians should consider that cholinesterase inhibitors may not be covered by provincial drug formularies for patients with a Mini-Mental State Examination score of less than 10. Also, memantine is covered only in Quebec, and only as monotherapy.

There are few data available to guide clinicians on when to discontinue therapy with cholinesterase inhibitors or memantine. The studies noted earlier included patients with a MiniMental State Examination score as low as 3-5, although patients with even lower scores might also benefit from these therapies. The consensus conference recommends, on the basis of level 3 evidence (expert opinion), that these drugs be continued until clinical benefit can no longer be determined. Patients who are bedridden, are noncommunicative and can no longer perform basic activities of daily living can usually have their medications safely discontinued.

In this progressive illness, besides improvement or stabilization of cognition, function and behaviour, even a slower than expected rate of decline can be considered a benefit of therapy. The best way to assess the benefits of therapy objectively might be to compare the rates of cognitive and functional decline before and after the start of therapy. This presumes that there would be repeated objective documentation

Box 5: Management of cognitive decline in patients with severe Alzheimer disease

- A cholinesterase inhibitor or memantine, or both, can be prescribed. Expected benefits include modest improvements, or a slower decline, in cognition, function and behaviour (grade A recommendation, level 1 evidence).

- The treatment should be continued until clinical benefit can no longer be demonstrated. It should not be stopped simply because the patient has been admitted to a long-term care facility (grade $\mathrm{C}$ recommendation, level 3 evidence). 
of global and cognitive measures of function. If the rate of decline appears to be more rapid after the start of therapy, discontinuing the drug would be appropriate. However, in some studies, patients who appeared initially to benefit from the therapy experienced rapid cognitive decline ${ }^{67}$ withdrawal symptoms ${ }^{68}$ and poorer prognosis (acute deterioration and death $)^{69}$ after cholinesterase inhibitors were stopped. Clinicians should therefore monitor patients closely after discontinuing these drugs because of the risks of significant withdrawal symptoms and cognitive decline.

\section{Knowledge gaps}

More research is required to clarify the definition of severe Alzheimer disease. At present, few studies assist clinicians in describing the disease trajectory as it progresses from mild to moderate to severe stages. Also, few data clearly identify prognostic factors that predict who will survive and who will maintain an adequate quality of life. Additional studies with clinically meaningful long-term outcomes are required to identify and support treatments aimed at delaying progression and managing symptoms. In terms of methodologic concerns, many studies that were reviewed for the guidelines included patients with moderate to severe Alzheimer disease. As a consequence, it was often impossible to separate the results for patients with only severe illness.

Patients with severe Alzheimer disease cannot complete the standardized cognitive assessments that have typically been used in the pivotal pharmacotherapy studies. Clinically useful measures of cognition for this stage of illness may assist in the day-to-day management of the disease, as well as assist researchers in defining effective interventions. Similarly, because patients are so dependent on caregivers, measures designed for this severe stage of disease would help in better describing clinically important benefits of therapy. This would be especially important for patients in long-term care facilities, who often do not have the opportunity to demonstrate elements of independence in many of the activities of daily living as a measure of treatment benefit. Although specific suggestions for trial design and outcome measures at this stage of illness have been made, ${ }^{70}$ the most significant concern would be to better define the magnitude of change considered clinically meaningful in assessing treatment benefits using many of the common rating scales..$^{71}$ Another significant limitation in developing recommendations for pharmacological interventions was the short duration (3-6 months) of most of the trials we reviewed. There were insufficient highquality trials to clearly define the optimal length of therapy with cholinesterase inhibitors and memantine and the appropriate approaches to discontinuation of the drugs.

Few high-quality trials of drug therapies for the behavioural and psychological symptoms that frequently accompany severe Alzheimer disease have been conducted to provide strong evidence for recommendations. We believe that safer and more effective drug therapy for agitation and aggression should be a significant research priority. Similarly, more effective nonpharmacologic approaches should be tested in rigorous randomized controlled trials.

\section{The case revisited}

Mr. L has reached the stage of severe Alzheimer disease. His family physician has been seeing him along with his wife every 3 months for the past 2 years. A year ago, the physician and Mrs. L agreed that the goals of management at this stage of the illness were to improve the quality of life for Mr. L, maintain optimal function and provide maximum comfort. With the help of the physician, Mrs. L arranges for home care and has modifications done at home to ensure it is safe for her husband. Because of his night-time wandering, the physician recommends the installation of special locks and an alarm system. He also recommends that Mr. L be registered on the local Alzheimer Society of Canada's Safely Home Registry. Mrs. L was referred to the local chapter of the Alzheimer Society of Canada several years earlier and continues to attend the support groups regularly.

To address the worsening agitation and aggressive behaviour, the physician completes a physical examination and orders laboratory tests for a complete blood count, measurement of serum electrolytes, calcium and fasting glucose, and a urinalysis. All of the findings are negative, and there is no evidence of delirium. The agitation and aggression are considered to be behavioural and psychological symptoms associated with progression of the Alzheimer disease. Before drug therapy is prescribed, the physician considers nonpharmacologic approaches. Mr. L has been attending a daycare program twice a week and has been sleeping better the nights he attends the program. The physician therefore suggests that he attend the daycare program 5 days per week.

To deal with Mr. L's problem of not recognizing himself in the mirror, the physician recommends that Mrs. L remove all of the mirrors in their home or spray them with fake snow. Because Mr. L appears to be much less resistive with male caregivers than with female caregivers, the physician asks Mrs. L to contact the home-care service to see if more male caregivers can be assigned. In addition, the physician suggests that Mrs. L play some of his favourite classical music during times of dressing and bathing, when he is most resistive.

$\mathrm{Mr}$. $\mathrm{L}$ is already receiving the maximal amount of homecare services available, yet Mrs. L spends most of her time caring for her husband. She mentions her increasing levels of stress and problems with insomnia. Upon direct questioning, she clearly states that she has no thoughts of suicide. Mrs. L is advised to see a social worker or psychologist to develop coping strategies. She is also told that her children should now be involved and should attend the next appointment.

Because Mr. L's psychosis, agitation and aggression are serious and represent a risk of harm for his wife, the physician considers further management with psychotropic drug therapy. After discussing the risks and benefits of atypical antipsychotics ${ }^{72}$ with Mrs. L, she agrees to a trial of medication.

Following a series of visits over 3 months, it appears that most of the recommended interventions have been helpful. Although Mr. L remains somewhat resistive to care, he has been calmer, sleeps better at night and has not experienced another aggressive episode. After 6 months of behavioural stability, his dose of antipsychotic drug is tapered and then stopped. 
Despite the improvement in behaviour, Mr. L continues to experience declines in cognitive and global function. His children have attended some of his appointments and provide their mother with some respite on weekends. They have expressed increasing concern about their mother's ability to deal emotionally and physically with the increasing demands for care of their father. With the whole family, the physician discusses the advantages and disadvantages of institutional care.

\section{Conclusion}

Severe Alzheimer disease represents a substantial societal burden because of its prevalence, costs and the suffering incurred by patients and their caregivers. The recommendations from the Third Canadian Consensus Conference on the Diagnosis and Treatment of Dementia, based on the best available evidence, have the potential to improve the quality of life of patients at this stage of disease as well as their caregivers. Ultimately, effective disease-modifying therapy for mild Alzheimer disease should hopefully preclude the need to treat the severe stage of illness. Additional research is warranted in order to improve the care of patients with this devastating disease.

\section{This article has been peer reviewed.}

Competing interests: Nathan Herrmann has received research support, honoraria and consulting fees from Lundbeck, Janssen-Ortho Inc., Pfizer, Novartis and Eli Lilly. Serge Gauthier has been an investigator and consultant for Lundbeck, Pfizer and Merz.

Contributors: Both of the authors made substantial contributions to the conception and design of the paper, as well as to the analysis and interpretation of data. They both drafted the article, revised it critically for important intellectual content and approved the final version to be published.

Editor's Note: The background papers with supporting evidence for the recommendations from the Third Canadian Consensus Conference on the Diagnosis and Treatment of Dementia were published in the October 2007 issue of Alzheimer's and Dementia and are available at www.alzheimersand dementia.org. These articles are also freely available at www.cccdtd.ca (through agreement with Elsevier).

\section{REFERENCES}

1. Canadian Study of Health and Aging. Study methods and prevalence of dementia. CMAJ 1994; 150:899-913.

2. Patterson CJ, Gauthier S, Bergman H, et al. Canadian Consensus Conference on Dementia: a physician's guide to using the recommendations. CMAJ 1999;160:1738-42.

3. Chertkow H. Diagnosis and treatment of dementia: introduction. Introducing a series based on the Third Canadian Consensus Conference on the Diagnosis and Treatment of Dementia. CMAJ 2008;178:316-21.

4. Reisberg B, Ferris SH, de Leon MJ, et al. The Global Deterioration Scale for assessment of primary degenerative dementia. Am J Psychiatry 1982;139:1136-9.

5. Reisberg B. Global measures: utility in defining and measuring treatment response in dementia. Int Psychogeriatr 2007;19:421-56.

6. Boller F, Verny M, Hugonot-Diener L, et al. Clinical features and assessment of severe dementia. A review. Eur J Neurol 2002;9:125-36.

7. Scherder E, Oosterman J, Swaab D, et al. Recent developments in pain in dementia. BMJ 2005;330:461-4.

8. Allen NH, Burns A, Newton V, et al. The effects of improving hearing in dementia. Age Ageing 2003;32:189-93.

9. Magri F, Borza A, del Vecchio S, et al. Nutritional assessment of demented patients: a descriptive study. Aging Clin Exp Res 2003;15:148-53.

10. Nandy s, Parsons S, Cryer C, et al. Development and preliminary examination of the predictive validity of the Falls Risk Assessment Tool (FRAT) for use in primary care. J Public Health 2004;26:138-43.

11. Bullock R. The needs of the caregiver in the long-term treatment of Alzheimer disease. Alzheimer Dis Assoc Disord 2004;18:S17-23.

12. Kettl P. Helping families with end-of-life care in Alzheimer's disease. J Clin Psychiatry 2007;68:445-50.

13. Mittelman MS, Roth DL, Coon DW, et al. Sustained benefit of supportive inter- vention for depressive symptoms in caregivers of patients with Alzheimer's disease. Am J Psychiatry 2004;161:850-6.

14. Knopman DS, Berg JD, Thomas R, et al. Nursing home placement is related to dementia progression: experience from a clinical trial. Alzheimer's Disease Cooperative Study. Neurology 1999;52:714-8.

15. Hébert R, Dubois MF, Wolfson C, et al. Factors associated with long-term institutionalization of older people with dementia: data from the Canadian Study of Health and Aging. J Gerontol A Biol Sci Med Sci 2001;56:M693-9.

16. Yaffe K, Fox P, Newcomer R, et al. Patient and caregiver characteristics and nursing home placement in patients with dementia. JAMA 2002;287:2090-7.

17. Lanctot KL, Bowles SK, Herrmann N, et al. Drugs mimicking dementia. Dementia symptoms associated with psychotropic drugs in institutionalised cognitively impaired patients. CNS Drugs 2000;14:381-90.

18. Feldman HH, Jacova C, Robillard A, et al. Diagnosis and treatment of dementia: 2. Diagnosis. CMAJ 2008; 178:825-36.

19. Livingston G, Johnston K, Katona C, et al. Systematic review of psychological approaches to the management of neuropsychiatric symptoms of dementia. Am J Psychiatry 2005;162:1996-2021.

20. Snowden M, Sato K, Roy-Byrne P. Assessment and treatment of nursing home residents with depression or behavioral symptoms associated with dementia: a review of the literature. J Am Geriatr Soc 2003;51:1305-17.

21. Verkaik R, van Weert JC, Francke AL. The effects of psychosocial methods on depressed, aggressive and apathetic behaviors of people with dementia: a systematic review. Int J Geriatr Psychiatry 2005;20:301-14.

22. Ayalon L, Gum AM, Feliciano L, et al. Effectiveness of nonpharmacological interventions for the management of neuropsychiatric symptoms in patients with dementia: a systematic review. Arch Intern Med 2006;166:2182-8.

23. Spira AP, Edelstein BA. Behavioral interventions for agitation in older adults with dementia: an evaluative review. Int Psychogeriatr 2006;18:195-225.

24. Teri L, Logsdon RG, Uomoto J, et al. Behavioral treatment of depression in dementia patients: a controlled clinical trial. J Gerontol B Psychol Sci Soc Sci 1997;52:159-66

25. Vink AC, Birks JS, Bruinsma MS, et al. Music therapy for people with dementia Cochrane Database Syst Rev 2006;(1)CD003477.

26. Holmes C, Knights A, Dean C, et al. Keep music live: music and the alleviation of apathy in dementia subjects. Int Psychogeriatr 2006;18:623-30.

27. Svansdottir HB, Snaedal J. Music therapy in moderate and severe dementia of Alzheimer's type: a case-control study. Int Psychogeriatr 2006;18:613-21.

28. Sink KM, Holden KF, Yaffe K. Pharmacological treatment of neuropsychiatric symptoms of dementia: a review of the evidence. JAMA 2005;293:596-608.

29. Wooltorton E. Risperidone (Risperdal): increased rate of cerebrovascular events in dementia trials. CMAJ 2002;167:1269-70.

30. Wooltorton E. Olanzapine (Zyprexa): increased incidence of cerebrovascular events in dementia trials. CMAJ 2004;170:1395.

31. Health Canada endorsed important safety information on atypical antipsychotic drugs and dementia: increased mortality associated with the use of atypical antipsychotic drugs in elderly patients with dementia [Dear Health Care Professional letter]. Ottawa $(\mathrm{ON})$ : Canadian Adverse Drug Reaction Monitoring Program, Health Canada; 2005. Available: www.hc-sc.gc.ca/dhp-mps/medeff/advisories-avis /prof/_2005/atyp-antipsycho_hpc-cps-eng.php (accessed 2008 Sept 25).

32. Herrmann N, Lanctot KL. Do atypical antipsychotics cause stroke? CNS Drugs 2005;19:91-103.

33. Gill SS, Rochon PA, Herrmann N, et al. Atypical antipsychotic drugs and risk of ischaemic stroke: population based retrospective cohort study. BMJ 2005;330:445.

34. Liperoti R, Gambassi G, Lapane KL, et al. Cerebrovascular events among elderly nursing home patients treated with conventional or atypical antipsychotics. $J$ Clin Psychiatry 2005;66:1090-6.

35. Schneider LS, Dagerman KS, Insel P. Risk of death with atypical antipsychotic drug treatment for dementia: meta-analysis of randomized placebo-controlled trials. JAMA 2005;294:1934-43.

36. Nasrallah HA, White T, Nasrallah AT. Lower mortality in geriatric patients receiving risperidone and olanzapine versus haloperidol: preliminary analysis of retrospective data. Am J Geriatr Psychiatry 2004;12:437-9.

37. Wang PS, Schneeweiss S, Avorn J, et al. Risk of death in elderly users of conventional vs. atypical antipsychotic medications. N Engl J Med 2005;353:2335-41.

38. Gill SS, Bronskill SE, Normand SL, et al. Antipsychotic drug use and mortality in older adults with dementia. Ann Intern Med 2007;146:775-86.

39. Schneider LS, Tariot PN, Dagerman KS, et al. Effectiveness of atypical antipsychotic drugs in patients with Alzheimer's disease. N Engl J Med 2006;355:1525-38.

40. Katz I, de Deyn PP, Mintzer J, et al. The efficacy and safety of risperidone in the treatment of psychosis of Alzheimer's disease and mixed dementia: a meta-analysis of 4 placebo-controlled clinical trials. Int J Geriatr Psychiatry 2007;22:475-84.

41. Van Reekum R, Clarke D, Conn D, et al. A randomized, placebo-controlled trial of the discontinuation of long-term antipsychotics in dementia. Int Psychogeriatr 2002; $14: 197-210$.

42. Ballard CG, Thomas A, Fossey J, et al. A 3-month, randomized, placebocontrolled, neuroleptic discontinuation study in 100 people with dementia: the neuropsychiatric inventory median cutoff is a predictor of clinical outcome. J Clin Psychiatry 2004;65:114-9.

43. Herrmann N, Lanctot KL. Pharmacological management of neuropsychiatric symptoms of Alzheimer disease. Can J Psychiatry 2007;52:630-46.

44. American Psychiatric Association. Practice guidelines for the treatment of patients with Alzheimer's disease and other dementias, Second Edition. Am J Psychiatry 2007; 164:1-56. 
45. Olin J, Schneider L, Katz I, et al. Provisional diagnostic criteria for depression of Alzheimer Disease. Am J Geriatr Psychiatry 2002;10:125-8.

46. American Psychiatric Association. Diagnostic and statistical manual of mental disorders. 4th ed, text revision. Washington (DC): American Psychiatric Press; 2000. p. $369-76$.

47. Thompson S, Herrmann N, Rapoport MJ, et al. Efficacy and safety of antidepressants for treatment of depression in Alzheimer's disease: a metaanalysis. Can J Psychiatry 2007;52:248-55.

48. Teri L, Gibbons LE, McCurry SM, et al. Exercise plus behavioral management in patients with Alzheimer disease: a randomized controlled trial. JAMA 2003;290: 2015-22.

49. Feldman H, Gauthier S, Hecker J, et al. A 24-week, randomized, double-blind study of donepezil in moderate to severe Alzheimer's disease. Neurology 2001;57: 613-20.

50. Tariot PN, Cummings JL, Katz IR, et al. A randomized, double-blind, placebocontrolled study of the efficacy and safety of donepezil in patients with Alzheimer's disease in the nursing home setting. J Am Geriatr Soc 2001:49:1590-9.

51. Bullock R, Touchon J, Bergman H, et al. Rivastigmine and donepezil treatment in moderate to moderately-severe Alzheimer's disease over a 2-year period. Curr Med Res Opin 2005;21:1317-27.

52. Winblad B, Kilander L, Eriksson S, et al. Donepezil in patients with severe Alzheimer's disease: double-blind, parallel-group, placebo-controlled study. Lancet 2006;367:1057-65.

53. Black SE, Doody R, Li H, et al. Donepezil preserves cognition and global function in patients with severe Alzheimer disease. Neurology 2007;69:459-69.

54. Birks J. Cholinesterase inhibitors for Alzheimer's disease. Cochrane Database Syst Rev 2006 Jan 25;(1):CD005593.

55. Winblad B, Poritis N. Memantine in severe dementia: results of the 9M-Best Study (Benefit and efficacy in severely demented patients during treatment with memantine). Int J Geriatr Psychiatry 1999;14:135-46.

56. Reisberg B, Doody R, Stoffler A, et al. Memantine in moderate-to-severe Alzheimer's disease. N Engl J Med 2003;348:1333-41.

57. Tariot PN, Farlow MR, Grossberg GT, et al. Memantine treatment in patients with moderate to severe Alzheimer disease already receiving donepezil: a randomized controlled trial. JAMA 2004;291:317-24.

58. Jones RW, Bayer A, Inglis F, et al. Safety and tolerability of once-daily versus twice-daily memantine: a randomised, double-blind study in moderate to severe Alzheimer's disease. Int J Geriatr Psychiatry 2007;22:258-62.

59. Winblad B, Jones RW, Wirth Y, et al. Memantine in moderate to severe Alzheimer's disease: a meta-analysis of randomised clinical trials. Dement Geriatr Cogn Disord 2007;24:20-7.

60. Wilkinson D, Andersen HF. Analysis of the effect of memantine in reducing the worsening of clinical symptoms in patients with moderate to severe Alzheimer's disease. Dement Geriatr Cogn Disord 2007;24:138-45.

61. Feldman HH, Schmitt FA, Olin JT. Activities of daily living in moderate-to-severe Alzheimer disease: an analysis of the treatment effects of memantine in patients receiving stable donepezil treatment. Alzheimer Dis Assoc Disord 2006;20:263-8.

62. Livingston G, Katona C. The place of memantine in the treatment of Alzheimer's disease: a number needed to treat analysis. Int J Geriatr Psychiatry 2004;19:919-25.

63. Gauthier S, Wirth Y, Mobius HJ. Effects of memantine on behavioural symptoms in Alzheimer's disease patients: an analysis of the Neuropsychiatric Inventory (NPI) data of two randomised, controlled studies. Int J Geriatr Psychiatry 2005; 20:459-64.

64. Ridha BH, Josephs KA, Rossor MN. Delusions and hallucinations in dementia with Lewy bodies: worsening with memantine. Neurology 2005;65:481-2.

65. Monastero R, Camarda C, Pipia C, et al. Visual hallucinations and agitation in
Alzheimer's disease due to memantine: report of three cases. J Neurol Neurosurg Psychiatry 2007;78:546. Epub 2006 Oct 9.

66. Gagnon M, Rive B, Hux M, et al. Cost-effectiveness of memantine compared with standard care in moderate-to-severe Alzheimer disease in Canada. Can J Psychiatry 2007;52:519-26.

67. Doody RS, Geldmacher DS, Gordon B, et al. Open-label, multicenter, phase 3 extension study of the safety and efficacy of donepezil in patients with Alzheimer disease. Arch Neurol 2001;58:427-33.

68. Singh S, Dudley C. Discontinuation syndrome following donepezil cessation. Int J Geriatr Psychiatry 2003;18:282-4.

69. Simpson S, Beavis D, Leddy A, et al. Naturalistic audit of NICE criteria for the use of cholinesterase inhibitors. Psychiatr Bull 2005;29:410-2.

70. Herrmann N. Treatment of moderate to severe Alzheimer's disease: rationale and trial designs. Can J Neurol Sci 2007;34:S103-8.

71. Hogan DB. Donepezil for severe Alzheimer's disease. Lancet 2006;367:1031-2.

72. Herrmann N, Lanctot KL. Atypical antipsychotics for neuropsychiatric symptoms of dementia: Malignant or maligned? Drug Saf 2006;29:833-43.

Correspondence to: Dr. Nathan Herrmann, Division of Geriatric Psychiatry, Sunnybrook Health Sciences Centre, Rm. FG05, 2075 Bayview Ave., Toronto ON M4N 3M5; fax 416 480-6022, n.herrmann@utoronto.ca

\section{Articles to date in this series}

- Chertkow H. Diagnosis and treatment of dementia: Introduction. Introducing a series based on the Third Canadian Consensus Conference on the Diagnosis and Treatment of Dementia. CMAJ 2008;178:316-21.

- Patterson C, Feightner JW, Garcia A, et al. Diagnosis and treatment of dementia: 1. Risk assessment and primary prevention of Alzheimer disease. CMAJ 2008;178:548-56.

- Feldman HH, Jacova C, Robillard A, et al. Diagnosis and treatment of dementia: 2. Diagnosis. CMAJ 2008;178:825-36.

- Chertkow H, Massoud F, Nasreddine Z, et al. Diagnosis and treatment of dementia: 3. Mild cognitive impairment and cognitive impairment without dementia. CMAJ 2008;178:1273-85.

- Hogan DB, Bailey P, Black S, et al. Diagnosis and treatment of dementia: 4. Approach to management of mild to moderate dementia. CMAJ 2008;179:787-93.

- Hogan DB, Bailey P, Black S, et al. Diagnosis and treatment of dementia: 5. Nonpharmacologic and pharmacologic therapy for mild to moderate dementia. CMAJ 2008;179: 1019-26. 